\title{
Intraocular pressure correlates with optic nerve sheath diameter in patients with normal tension glaucoma
}

\author{
Luís Abegão Pinto • Evelien Vandewalle • Anna Pronk • \\ Ingeborg Stalmans
}

Received: 20 September 2011 /Revised: 8 November 2011 / Accepted: 15 November 2011 /Published online: 10 December 2011

(C) Springer-Verlag 2011

\begin{abstract}
Purpose 1. Identify differences in optic nerve sheath diameter (ONSD) as an indirect measure of intracranial pressure (ICP) in glaucoma patients and a healthy population. 2. Identify variables that may correlate with ONSD in primary open-angle glaucoma (POAG) and normal tension glaucoma (NTG) patients.

Methods Patients with NTG $(n=46)$ and POAG $(n=61)$, and healthy controls $(n=42)$ underwent B-scan ultrasound measurement of ONSD by an observer masked to the patient diagnosis. Intraocular pressure (IOP) was measured in all groups, with additional central corneal thickness (CCT) and visual field defect measurements in glaucomatous patients. Only one eye per patient was selected. Kruskal-Wallis or Mann-Whitney were used to compare the different variables between the diagnostic groups. Spearman correlations were used to explore relationships among these variables.

Results ONSD was not significantly different between healthy, NTG and POAG patients $(6.09 \pm 0.78,6.03 \pm 0.69$,
\end{abstract}

\footnotetext{
L. Abegão Pinto

Department of Ophthalmology,

Centro Hospitalar de Lisboa Central,

Alameda de Santo António dos Capuchos,

1169-050 Lisbon, Portugal

L. Abegão Pinto

Institute of Pharmacology and Neurosciences,

Faculty of Medicine, Lisbon University,

Av. Professor Egas Moniz,

1649-028 Lisbon, Portugal

E. Vandewalle $\cdot$ A. Pronk $\cdot$ I. Stalmans $(\bowtie)$

Department of Ophthalmology,

University Hospitals Leuven, Belgium,

Campus St Raphael, Kapucijnenvoer 33,

B-3000 Leuven, Belgium

e-mail: ingeborg.stalmans@uzleuven.be
}

and $5.71 \pm 0.83$ respectively; $p=0.08$ ). Visual field damage and CCT were not correlated with ONSD in either of the glaucoma groups (POAG, $p=0.31$ and 0.44 ; NG, $p=0.48$ and 0.90 respectively). However, ONSD did correlate with IOP in NTG patients $(r=0.53, p<0.001)$, while it did not in POAG patients and healthy controls $(p=0.86, p=0.46$ respectively). Patient's age did not relate to ONSD in any of the groups ( $p>0.25$ in all groups).

Conclusions Indirect measurements of ICP by ultrasound assessment of the ONSD may provide further insights into the retrolaminar pressure component in glaucoma. The correlation of ONSD with IOP solely in NTG patients suggests that the translaminar pressure gradient may be of particular importance in this type of glaucoma.

Keywords Optic nerve sheath · Intracranial pressure . Glaucoma $\cdot$ Translaminar gradient

\section{Introduction}

Glaucoma is an optic neuropathy defined by a characteristic structural damage at the optic nerve head (ONH). Although lowering intraocular pressure (IOP) helps to slow down or stabilize the disease, a significant number of patients still show signs of progression despite an IOP within the normal range. Extensive research has therefore extended into nonIOP related risk factors. In animal models, one variable that has been shown to be related to structural changes in the $\mathrm{ONH}$ similar to the glaucomatous damage is intracranial pressure (ICP) [1]. The literature suggests that pressure gradients between intra- and extra-ocular compartments may be involved in the damage to the retinal ganglion cell's axons that cross the lamina cribosa $[2,3]$. However, the need for painful and invasive techniques to study ICP, such 
as lumbar puncture, has deterred most authors from evaluating this variable in the overall glaucoma research field.

Ultrasound-based assessment of the optic nerve sheath diameter (ONSD) is a validated method for indirect measurement of the ICP [4-6]. The optic nerve (ON) is separated from its sheath by a fluid layer of cerebral spinal fluid (CSF), which is in continuity with the rest of the central nervous system. Increases in ICP lead to an increase in the volume of the fluid layer, thereby increasing ONSD as measured in the retrobulbar portion of the ON trajectory. Ultrasound-based ONSD measurements have been extensively used to quickly and non-invasively assess ICP both at clinical and research levels. From medical emergency screening for changes in ICP in intracranial hemorrhages [7] to clinical research in ICP variations to mountain climbing [8], a vast literature now exists validating the use of this method $[5,6]$. Nevertheless, and to the best of our knowledge, no study has yet used ultrasound measurements of ONSD to investigate ICP significance in glaucoma.

Our study aims at identifying differences in ONSD between glaucoma and a healthy population. Furthermore, we studied variables that may be associated with these indirect ICP measurements.

\section{Methods}

\section{Subject groups}

Three cohorts of individuals over 18 years old were recruited for the study in the University Hospitals of Leuven: patients with NTG ( $n=46)$, patients with POAG $(n=61)$, and healthy control subjects $(n=42)$. This latter group was recruited from the cataract surgery clinic. Glaucoma patients were defined as having characteristic optic disc damage and visual field loss as described previously in the literature $[9,10]$. For the diagnosis of POAG, an untreated IOP of $21 \mathrm{mmHg}$ or above was required. Current medical treatment, including topical IOP-lowering drugs, was continued. The healthy volunteers were screened by a senior member of the glaucoma clinic (IST) and those with a family history of glaucoma, an increased or asymmetrical cup/disc ratio or any other optic disc structural change (notching, disc hemorrhage), or an IOP above $21 \mathrm{mmHg}$, were excluded as possible glaucoma suspects. Patients with a history of ocular trauma or eye disease (except glaucoma) that could not be accounted for by refractive error were excluded. Any known neurological disorder was also an exclusion criterion.

The study was approved by the ethical review committee (Institutional Review Board) at the University Hospitals Leuven, and was conducted in accordance with Good Clinical Practice within the tenets of the Helsinki agreement. Each patient/subject was required to sign an informed consent statement before being enrolled into the study and prior to any study measurements being taken.

\section{Measuring devices}

IOP was measured with the Goldmann applanation tonometer (GAT). Central corneal thickness (CCT) was measured using a Pachmate DGH55 (DGH Technology Inc., Exton, PA, USA). Measurement of the ONSD was performed with a B-scan ultrasound probe (Antares ecograph device, Siemens, Munich, Germany).

\section{Experimental design}

Patients were instructed to avoid caffeine intake, smoking, and exercise for 3 hours prior to the study visit. During the study visit, the following examinations were performed in the same order: visual acuity (using the ETDRS chart placed in the same location at the same distance from the patient under the same illumination for all subjects), IOP measurement by GAT, pachymetry, blood pressure and heart rate measurements, and finally ONSD measurements. This latter was performed by an observer (LAP) masked to the patient diagnosis, using the methodology described in the literature [4]: The patient lies in the supine position with the head in a neutral position and both eyes closed and in primary gaze position. After coupling gel is applied and the insonation depth is set to $5-8 \mathrm{~cm}$, the transducer is softly placed over the upper eyelid in an axial plane. This sonographic section provides a transverse view of the globe and the structures of the retrobulbar area (Fig. 1). The ONSD is calculated perpendicular to the vertical axis of the scanning place $3 \mathrm{~mm}$ behind the globe, where the $\mathrm{ON}$ sheath structure is more prone to expansion due to increases in ICP [11], probably

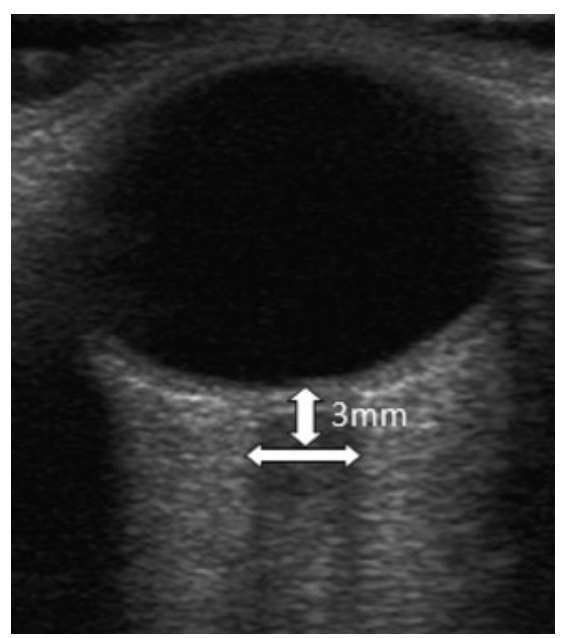

Fig. 1 Optic nerve sonography. The optic nerve complex is shown as a sharply-defined hypoechoic stripe in between the echogenic retrobulbar fat 
due to a decrease in sheath thickness in that retrobulbar segment of the ON $[12,13]$.

Data regarding axial length as measured by ultrasonic biometry was retrieved from the patient's file. Information regarding functional and structural damage from glaucoma patients was collected from exams undertaken on the day of the study visit.

Only one eye per patient was included in the study. The eye with greater glaucomatous damage was selected in the glaucoma patients. In healthy individuals, the eye not submitted to cataract surgery was selected.

\section{Statistical analysis}

The Kruskal-Wallis test was used to compare between variables from the three diagnostic groups. The Mann-Whitney test was performed to compare between two variables. Spearman correlation was used to study associations between variables. Probabilities are two-sided and considered statistically significant if $p<0.05$ (Graphpad Prism ${ }^{\circledR}$ ver. 5.0; Graphpad Software Inc, La Jolla, CA, USA). Descriptive results are reported as mean $\pm \mathrm{SD}$ unless otherwise indicated.

\section{Results}

Patient characteristics

Table 1 summarizes the patient characteristics in the different diagnostic groups with their comparative $p$ values. There

Table 1 Patient characteristics

\begin{tabular}{lllll}
\hline & Healthy & NTG & POAG & $\begin{array}{l}\text { Kruskal- } \\
\text { Wallis/ } \\
\text { Mann- } \\
\text { Whitney }\end{array}$ \\
\hline$N$ & 42 & 46 & 61 & \\
Age & $73.5 \pm 13.0$ & $65.4 \pm 13.8$ & $66.7 \pm 13.6$ & 0.004 \\
IOP & $14.0 \pm 3.3$ & $12.8 \pm 2.8$ & $14.4 \pm 5.6$ & 0.29 \\
Pachymetry & $558.3 \pm 26.6$ & $548.3 \pm 30.2$ & $557.2 \pm 34.0$ & 0.58 \\
Axial length & $22.0 \pm 1.3$ & $22.1 \pm 1.5$ & $22.4 \pm 2.0$ & 0.78 \\
MD & - & $-7.29 \pm 8.0$ & $-9.70 \pm 8.7$ & 0.11 \\
RNFL thickness & - & $0.17 \pm 0.12$ & $0.17 \pm 0.10$ & 0.76 \\
Visual acuity & $0.36 \pm 0.34$ & $0.28 \pm 0.33$ & $0.24 \pm 0.29$ & 0.48 \\
MOPP & $96.3 \pm 13.6$ & $97.3 \pm 14.7$ & $95.3 \pm 14.5$ & 0.78 \\
\hline
\end{tabular}

Mean values (and SD) are depicted. Kruskal-Wallis indicates $p$ values of overall differences between the three diagnostic groups. MannWhitney was used to compare variables between only two groups. $N=$ number of patients, $\mathrm{NTG}=$ normal tension glaucoma, POAG: primary open-angle glaucoma, IOP: intraocular pressure, MD: mean defect, RFNL: retinal nerve fiber layer thickness, BP: blood pressure, MOPP, median ocular perfusion pressure $[(2 / 3$ diastolic $+1 / 3$ systolic BPs) - Goldmann tonometry] were no differences between IOP records of the diagnostic groups $(p=0.29)$, nor between median ocular perfusion pressure (MOPP) measurements $(p=0.78)$. ONSD in the different diagnostic groups was not significantly different $(p=0.08)$. However, pairwise comparisons revealed $p$ values close to significance both when comparing between healthy individuals and POAG $(p=0.056)$ and between NTG and POAG patients $(p=0.07)$. Healthy controls versus NTG patients were not significantly different $(p=0.91)$. When comparing CCT, MD and retinal nerve fiber layer (RNFL) between the NTG and POAG patients, no statistical difference was determined ( $p=0.35 ; p=0.11 ; p=0.76$ respectively). Axial length was not different between any of the three groups $(p=0.78)$. Topical medications are summarized in Table 2 .

Relationship between ONSD and clinical variables from diagnostic groups

A positive correlation between ONSD and IOP was observed in the NTG group ( $p=0.0006 ; r=0.53$ ) (Fig. 2), while no such association was identified in the other groups (healthy: $p=0.46$; POAG: $p=0.86$ ). ONSD was not related to age in any of the diagnostic groups $(p>0.25)$. The axial length was also not correlated with ONSD $(p>0.15)$. Likewise, MD and CCT were also not significantly associated with ONSD in either of the glaucoma groups $(p>0.30)$. However, in the NTG group, we detected a near statistically significant association between the RNFL and the ONSD $(p=0.08)$. (Table 3).

\section{Discussion}

This study was conducted to evaluate the relevance of studying ONSD in glaucoma patients, and to study whether this indirect measurement of ICP correlates with any clinical variable in these patients.

In our study, no statistically significant differences in ONSD between glaucoma groups and control subjects could be demonstrated. As an indirect measure of ICP, our results

Table 2 Topical medications

\begin{tabular}{lll}
\hline & NTG & POAG \\
\hline Beta blockers & $19(41.3 \%)$ & $29(47.5 \%)$ \\
Prostaglandin analogs & $22(47.8 \%)$ & $34(55.7 \%)$ \\
Carbonic anhydrase inhibitors & $18(39.1 \%)$ & $16(26.2 \%)$ \\
$\alpha$-adrenergic agents & $5(10.9 \%)$ & $5(8.2 \%)$ \\
\hline
\end{tabular}

Number of patients and percentage (between brackets) are depicted. NTG: normal tension glaucoma; POAG: primary open-angle glaucoma 


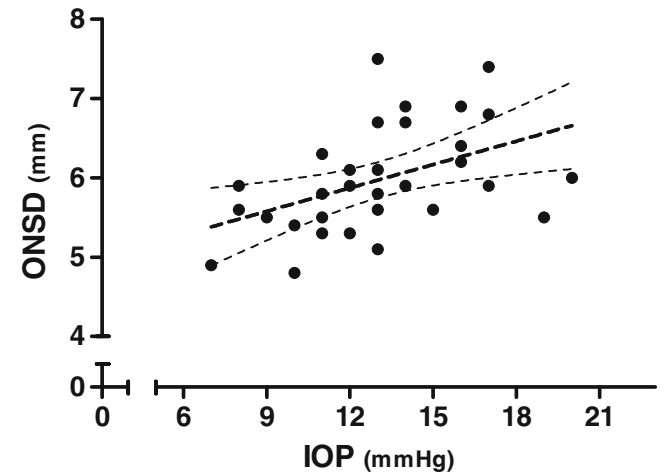

Fig. 2 Correlation between OSND and IOP in NTG group ( $p=0.0006$; $r=0.53$ ). Non-linear regression and $95 \%$ confidence interval is depicted $\left(\mathrm{Y}=4.690+0.109 \mathrm{X} ; r^{2}=0.20\right)$. OSND — optic nerve sheath diameter, $I O P$ - intraocular pressure

would suggest no significant difference in ICP exists between the groups. However, recent research has raised interesting questions as to whether glaucoma populations, specifically NTG individuals, are good candidates for this well-established ICP indirect measurement. In a recent study by Jaggi [14], an MRI-based measurement of the ONSD in NTG patients found an increased ONSD when compared to patients with other neurological disorders, suggesting this enlargement would not match the expected lower lumbar puncture-measured ICP in this population. These different results when compared to our data could have several explanations. One possibility for these authors' findings that NTG patients could have a larger ONSD than controls could relate to the methodology used. Not only could the invasiveness of $10 \mathrm{mM}$ iopamidol have interfered with the normal CSF circulation, but more importantly, there may be a difference in head position scanning between these two groups. Prone position has been demonstrated to influence CSF distribution in the central nervous system $[15,16]$. By

Table 3 Correlation between ONSD and variables from diagnostic groups

\begin{tabular}{|c|c|c|c|c|c|c|}
\hline & \multicolumn{2}{|c|}{ Healthy } & \multicolumn{2}{|l|}{ NTG } & \multicolumn{2}{|c|}{ POAG } \\
\hline & $P$ & $R$ & $P$ & $R$ & $P$ & $R$ \\
\hline Age & 0.50 & -0.10 & 0.75 & -0.005 & 0.26 & 0.16 \\
\hline MD & - & - & 0.48 & 0.11 & 0.31 & -0.14 \\
\hline RNFL & - & - & 0.08 & -0.31 & 0.70 & -0.05 \\
\hline $\mathrm{CCT}$ & - & - & 0.90 & -0.001 & 0.44 & -0.11 \\
\hline IOP & 0.46 & -0.08 & 0.0006 & 0.53 & 0.86 & -0.02 \\
\hline Axial length & 0.17 & 0.25 & 0.56 & -0.11 & 0.54 & -0.09 \\
\hline
\end{tabular}

Spearman correlations are depicted [values on coefficient of correlation $(r)$ and $p$ values $(p)]$. MD - mean defect; RNFL - retinal nerve fiber layer; IOP — intraocular pressure; CCT - central corneal thickness; NTG - normal tension glaucoma; POAG - primary open-angle glaucoma; ONSD - optic nerve sheath diameter measuring NTG patients in a prone position, there is a possibility that the gravitational change in CSF distribution could have changed the volume inside the ON sheaths, thus interfering in the results. Our prospective study, while being completely non-invasive, used the same method of ONSD measurement in all patients. Additionally, the measurement location and resolution of both imaging techniques could also explain the different results. The MRI reconstruction images are based on 32 sections of $0.5 \mathrm{~mm}$ with data analysis from reconstruction slices of $1 \mathrm{~mm}$ thickness, while the average resolution of a 7-12 MHz ultrasound is between 130 and $800 \mu \mathrm{m}$ [17]. Moreover, we have tested our measurements at the same position in all the patients, while the MRI study searched for the wider ONSD section area.

Data from both retrospective and prospective studies suggest that peripherally measured ICP is diminished in NTG patients when compared to other neurologic patients $[18,19]$. While there is a possibility for the underlying neurological condition to have an impact on the ICP, one would expect ONSD to be decreased in such patients. While the nature of such a decrease in ICP is still not understood, that decrease would aggravate the translaminar gradient for an otherwise normal IOP. Nevertheless, existing data suggests that possibly in these patients, orbital ICP may not exactly reflect the supine, lumbar-measured ICP. Data from direct contrast-injection into the subarachnoid space of glaucoma patients have proven that CSF fluid dynamics are disturbed in the retrobulbar part of the optic nerve sheath [20].

As our results do not show a difference between these populations in ONSD, we can assume that the summation of the forces acting behind the lamina cribosa is not statistically different (at least within the limits of ultrasound resolution). This, however, does not necessarily mean that these resulting forces would have a similar impact on the optic nerve. Indeed, the translaminar gradient is not only affected by the IOP and the retrolaminar pressure, but also by the thickness of the wall separating these compartments. An abnormally thin sclera, as suggested to exist in NTG patients [21], could dramatically increase the pressure gradient over this supporting tissue [22], even with pressures in the retroocular compartments within an otherwise normal range. This could then result in an increased stress on the optic nerve in these patients.

One interesting aspect of our results is that the ONSD did not correlate with age. Published data suggests there is a decrease on the neuron mass of the optic nerve with advancing age and in more advanced glaucoma [23, 24]. The fact that the overall ONSD is unchanged with age and glaucoma damage would imply that the ratio between the neuron mass and the CSF inside the ONSD is a dynamic one. This would help explain part of the difficulties in studying the retrobulbar component in glaucoma pathogenesis. 
Interestingly, the ONSD measurement had a positive correlation with IOP in NTG patients, while it did not in any of the other groups. As it seems unlikely that the transcorneal gradient pressure (IOP) as measured by Goldmann applanation tonometry could affect the forces acting behind the lamina cribosa [25], there is a possibility that those forces may affect IOP by correlating with either aqueous humor (AH) production or drainage. There is evidence that ICP may play a significant role in $\mathrm{AH}$ drainage by modulating ocular venous drainage [26]. According to the Monro-Kellie doctrine, intracranial blood volumes vary reciprocally with changes in the CSF volume [27]. Accordingly, a decrease or increase in CSF volume directly or indirectly influences the flow inside the intracranial venous sinuses, and therefore influences the drainage of the superior ophthalmic veins (SOVs). These changes in intracranial hydrodynamics may explain why increased levels of ICP lead to a significant dilation of this SOVs $[28,29]$. As these SOVs are responsible for most of the ocular venous drainage, including the $\mathrm{AH}$ drainage-related anterior ciliary veins, there is a possibility of these changes having an impact in IOP. An extreme example of this ability to influence IOP is the secondary open-angle glaucoma caused by thrombosis of the SOV [30]. Accordingly, one possibility for the observed ONSD/ IOP correlation in NTG patients is that any change in downstream gradients will lead to a similar change in drainage of AH. NTG patients may have some unknown structural weaknesses along the extra-ocular drainage pathway (in venous tonus, for instance) that may render them incapable to significantly buffer any change in the forces acting outside the globe. In fact, venous circulation in glaucoma patients - and specially NTG patients - has been proved to behave differently than in healthy individuals, ranging from different peripapilary vessel diameter [31] and rigidity [32] to significant decrease in prevalence of spontaneous venous phenomenon [33, 34]. The degree of the impact of this vascular venous dysregulation on IOP and the extent to which it would interfere with the drainage of an otherwise normal IOP on NTG is not fully understood. However, and since the pressure inside that venous compartment has to be maintained at a lower level than IOP for AH drainage to occur, we can hypothesize that at lower levels of ICP, the other forces acting in the orbit could interfere with the SOV drainage. Hypothetically, the existence of a greater upstream obstruction on the drainage pathway in POAG patients, such as in the trabecular meshwork for example, might explain why this ONSD would not have correlated with IOP.

Our work has several limitations. As a full neurological examination was not performed in these patients, there is the possibility of a contamination of the experimental groups by individuals with a yet undiagnosed neurological clinical condition. Furthermore, the refractive status of the patients could also introduce a bias, as the properties of the sclera are different from hyperopic to myopic patients [35]. As some patients were pseudophakic, we assessed the axial length, which was inside the normal range and similar in all groups. More importantly, the fitness of the linear regression model explaining the ONSD/IOP relationship in NTG patients is low, despite a higher level of correlation between the ONSD and IOP variables. This would suggest there may be a nonlinear correlation with these parameters, with further studies being needed in order to understand the nature of this relationship.

In summary, orbital imaging techniques, such as noninvasive ultrasound-based ONSD assessment, may provide further insights into the forces acting behind the lamina cribosa. More studies will need to further investigate the intriguing relationship between ICP and the optic nerve, whose significance in glaucoma may of particular importance in NTG patients.

Acknowledgements The authors would like to thank Veerle Vanbellinghen and Sien Boons for their assistance with the examinations of the patients.

\section{References}

1. Yablonski M, Ritch R, Pokorny KS (1979) Effect of decreased intracranial pressure on optic disc. Invest Ophthalmol Vis Sci 18: S165

2. Jonas JB, Berenshtein E, Holbach L (2003) Anatomic relationship between lamina cribrosa, intraocular space, and cerebrospinal fluid space. Invest Ophthalmol Vis Sci 44:5189-5195

3. Morgan WH, Yu DY, Balaratnasingam C (2008) The role of cerebrospinal fluid pressure in glaucoma pathophysiology: the dark side of the optic disc. J Glaucoma 17:408-413

4. Soldatos T, Chatzimichail K, Papathanasiou M (2009) Optic nerve sonography: a new window for the non-invasive evaluation of intracranial pressure in brain injury. Emerg Med J 26:630-634

5. Moretti R, Pizzi B, Cassini F, Vivaldi N (2009) Reliability of optic nerve ultrasound for the evaluation of patients with spontaneous intracranial hemorrhage. Neurocrit Care 11:406-410

6. Bäuerle J, Lochner P, Kaps M, Nedelmann M (2010) Intra- and interobserver reliability of sonographic assessment of the optic nerve sheath diameter in healthy adults. J Neuroimaging Dec 1 [Epub ahead of print]. doi:10.1111/j.1552-6569.2010.00546

7. Tayal VS, Neulander M, Norton HJ (2007) Emergency department sonographic measurement of optic nerve sheath diameter to detect findings of increased intracranial pressure in adult head injury patients. Ann Emerg Med 49:508-514

8. Sutherland AI, Morris DS, Owen CG, Bron AJ, Roach RC (2008) Optic nerve sheath diameter, intracranial pressure and acute mountain sickness on Mount Everest: a longitudinal cohort study. Br J Sports Med 42:183-188

9. Jampel H (1997) Target pressure in glaucoma therapy. J Glaucoma 6:133-138

10. Zeyen T (1999) Target pressures in glaucoma. Bull Soc Belge Ophtalmol 274:61-65

11. Liu D, Kahn M (1993) Measurement and relationship of subarachnoid pressure of the optic nerve to intracranial pressures in fresh cadavers. Am J Ophthalmol 116:548-556 
12. Hayreh SS (1968) Pathogenesis of oedema of the optic disc. Doc Ophthalmol 24:289-411

13. Hayreh SS (1984) The sheath of the optic nerve. Ophthalmologica 189:54-63

14. Jaggi GP, Miller NR, Flammer J, Weinreb RN, Remonda L, Killer HE (2011) Optic nerve sheath diameter in normal-tension glaucoma patients. Br J Ophthalmol Mar 11 [Epub ahead of print]. doi:10.1136/bjo.2010.199224

15. Yamada S, Miyazaki M, Kanazawa H, Higashi M, Morohoshi Y, Bluml S, McComb JG (2008) Visualization of cerebrospinal fluid movement with spin labeling at MR imaging: preliminary results in normal and pathophysiologic conditions. Radiology 249 (2):644-652

16. Lotz PR (1982) Intracranial delivery of metrizamide from the lumbar subarachnoid space: prone versus supine positioning. $\mathrm{J}$ Comput Assist Tomogr 6:920-922

17. Restori M (2008) Imaging the vitreous: optical coherence tomography and ultrasound imaging. Eye 22:1251-1256

18. Killer HE, Jaggi GP, Flammer J, Miller NR, Huber AR, Mironov A (2007) Cerebrospinal fluid dynamics between the intracranial and the subarachnoid space of the optic nerve. Is it always bidirectional? Brain 130:514-520

19. Berdahl JP, Fautsch MP, Stinnett SS, Allingham RR (2008) Intracranial pressure in primary open-angle glaucoma, normal tension glaucoma, and ocular hypertension: a case-control study. Invest Ophthalmol Vis Sci 49: 5412-5418

20. Ren R, Jonas JB, Tian G, Zhen Y, Ma K, Li S, Wang H, Li B, Zhang X, Wang N (2010) Cerebrospinal fluid pressure in glaucoma: a prospective study. Ophthalmology 117:259-266

21. Mohamed-Noor J, Bochmann F, Siddiqui MA, Atta HR, Leslie T, Maharajan P, Wong YM, Azuara-Blanco A (2009) Correlation between corneal and scleral thickness in glaucoma. J Glaucoma 18:32-36

22. Jonas JB, Berenshtein E, Holbach L (2004) Lamina cribrosa thickness and spatial relationships between intraocular space and cerebrospinal fluid space in highly myopic eyes. Invest Ophthalmol Vis Sci 45:2660-2665

23. Jonas JB, Schmidt AM, Muller-Bergh JA, Schldrzer-Schrehardr UM (1992) Human optic nerve fiber count and optic disc size. Invest Ophthalmol Vis Sci 33:2012-2018
24. Yücel YH, Gupta N, Kalichman MW, Mizisin AP, Hare W, de Souza LM, Zangwill L, Weinreb RN (1998) Relationship of optic disc topography to optic nerve fiber number in glaucoma. Arch Ophthalmol 116:493-497

25. Ren R, Wang N, Zhang X, Cui T, Jonas JB (2001) Trans-lamina cribrosa pressure difference correlated with neuroretinal rim area in glaucoma. Graefes Arch Clin Exp Ophthalmol 249:1057-1063

26. Spentzas T, Henricksen J, Patters AB, Chaum E (2010) Correlation of intraocular pressure with intracranial pressure in children with severe head injuries. Pediatr Crit Care Med 11:593-598

27. Mokri B (2001) The Monro-Kellie hypothesis: applications in CSF volume depletion. Neurology 56:1746-1748

28. Lirng JF, Fuh JL, Wu ZA, Lu SR, Wang SJ (2003) Diameter of the superior ophthalmic vein in relation to intracranial pressure. Am J Neuroradiol 24:700-703

29. Khanna RK, Pham CJ, Malik GM, Spickler EM, Mehta B, Rosenblum ML (1997) Bilateral superior ophthalmic vein enlargement associated with diffuse cerebral swelling. Report of 11 cases. J Neurosurg 86:893-897

30. Foroozan R, Buono LM, Savino PJ, Sergott RC (2003) Idiopathic dilated episcleral veins and increased intraocular pressure. $\mathrm{Br} \mathrm{J}$ Ophthalmol 87:652-654

31. Jonas JB, Nguyen XN, Naumann GO (1989) Parapapillary retinal vessel diameter in normal and glaucoma eyes. I. Morphometric data. Invest Ophthalmol Vis Sci 30:1599-1603

32. Oettli A, Gugleta K, Kochkorov A, Katamay R, Flammer J, Orgul S (2011) Rigidity of retinal vessel in untreated eyes of normal tension primary open-angle glaucoma patients. J Glaucoma 20:303-306

33. Morgan WH, Hazelton ML, Azar SL, House PH, Yu DY, Cringle SJ, Balaratnasingam C (2004) Retinal venous pulsation in glaucoma and glaucoma suspects. Ophthalmology 111:1489-1494

34. Graham SL, Butlin M, Lee M, Ayolio AP (2011) Central blood pressure, arterial waveform analysis, and vascular risk factors in glaucoma. J Glaucoma Jun 28 [Epub ahead of print: PMID: 21716126]

35. Norman RE, Flanagan JG, Rausch SM, Sigal IA, Tertinegg I, Eilaghi A, Portnoy S, Sled JG, Ethier CR (2010) Dimensions of the human sclera: thickness measurement and regional changes with axial length. Exp Eye Res 90:277-284 